\title{
Compact 4-manifolds admitting special handle decompositions
}

\author{
Maria Rita Casali ${ }^{1}$ And PaOla Cristofori ${ }^{2}$ \\ ${ }^{1}$ Department of Physics, Mathematics and Computer Science, University of Modena and Reggio Emilia \\ ViaCampi213 B, I-41125 Modena (Italy), casali@unimore.it \\ ${ }^{2}$ Department of Physics, Mathematics and Computer Science, University of Modena and Reggio Emilia, \\ Via Campi 213 B, I-41125 Modena (Italy), paola.cristofori@unimore.it
}

August 27, 2020

\begin{abstract}
In this paper we study colored triangulations of compact PL 4-manifolds with empty or connected boundary which induce handle decompositions lacking in 1-handles or in 1- and 3-handles, thus facing also the problem, posed by Kirby, of the existence of special handlebody decompositions for any simply-connected closed PL 4-manifold. In particular, we detect a class of compact simplyconnected PL 4-manifolds with empty or connected boundary, which admit such decompositions and, therefore, can be represented by (undotted) framed links. Moreover, this class includes any compact simply-connected PL 4-manifold with empty or connected boundary having colored triangulations that minimize the combinatorially defined PL invariant regular genus, gem-complexity or G-degree among all such manifolds with the same second Betti number.
\end{abstract}

Keywords: compact 4-manifold, colored triangulation, handle decomposition, framed link, crystallization, regular genus.

2020 Mathematics Subject Classification: 57K40, 57M15, 57K10, 57Q15.

\section{Introduction}

The question of whether or not any simply-connected closed PL 4-manifold admits a handle decomposition lacking in 1-handles and 3-handles (i.e. a special handlebody decomposition, according to [30, Section 3.3]) is long standing: it first appears as problem n. 50 in Kirby's list of problems in low dimensional manifold topology, edited in 1977 (27]), it is reported unaltered as Problem 4.18 in the updated list by the same author eighteen years later ([28]), and so far Kirby's comment about "no progress" continues to be valid.

Actually, Kirby's problem can be formulated also with an intermediate weaker requirement:

"Does every simply-connected closed 4-manifold have a handlebody decomposition without 1-handles?

Without 1- and 3-handles?"

Significant investigations on the matter have been performed during the decades, taking into account simply-connected PL 4-manifolds with connected boundary, too: recall, for example, the important Trace's contributions [32] and [33. In the boundary case, it is known that each contractible 4-manifold different from $\mathbb{D}^{4}$ must have 1- or 3-handles, while a contractible 4-manifold requiring 1handles in any handle decomposition was detected by Casson (see [28, Problem 4.18]). As regards closed 4-manifolds, the so called Dolgachev surface $E(1)_{2,3}$, exotic copy of $\mathbb{C P}^{2} \#_{9} \overline{\mathbb{C P}^{2}}$, has long been 
conjectured to be a possible counterexample to the universality of special handlebody decompositions ([25]); however, Akbulut not only proved $E(1)_{2,3}$ to admit a handle decomposition lacking in 1-handles and 3-handles ([1]), but also produced an infinite family of exotic copies of $\mathbb{C P}^{2} \#_{9} \overline{\mathbb{C P}^{2}}$ with the same property $([2])$.

The present paper studies both questions posed by the above Kirby's problem, also in the extended setting of compact 4-manifolds with connected boundary, by making use of the so called colored triangulations (i.e. pseudosimplicial triangulations whose 4-simplices have vertices injectively labelled by five colors), together with their visualizing tool consisting of edge-colored graphs.

More precisely, a large class of colored triangulations (or, equivalently, of associated 5-colored graphs), possibly representing all simply-connected closed PL 4-manifolds, is identified, so that the induced handle decompositions turn out to be special.

From the point of view of colored triangulations, it is well known that any compact PL 4-manifold with empty or connected boundary admits a contracted triangulation, i.e. a colored triangulation with exactly five vertices, labelled by the five elements of the color set $\Delta_{4}=\{0,1,2,3,4\} 1$. In the closed setting, the main result of the present paper - involving both steps of the quoted Kirby's problem - is the following:

Theorem 1 Let $M^{4}$ be a closed PL 4-manifold and let $K$ be a contracted triangulation of $M^{4}$.

(a) If $K$ contains exactly one edge between two pairs of vertices, involving three vertices, then $M^{4}$ admits a handle decomposition without 1-handles;

(b) if $K$ contains exactly one edge between three pairs of vertices, involving all five vertices, then $M^{4}$ admits a special handlebody decomposition.

In the connected boundary case, by a contracted triangulation of a compact 4-manifold $M^{4}$ we mean a contracted triangulation of the associated singular 4-manifold $\widehat{M^{4}}$ obtained from $M^{4}$ by capping off the boundary $\partial M^{4}$ with a cone; moreover, color 4 is usually assumed to be singular, i.e. to label the only vertex whose link is a closed connected 3-manifold different from the 3 -sphere $\mathbb{S}^{3}$ (namely, the boundary $\partial M^{4}$, which is supposed to be non-spherical). In this setting, we prove the following result:

Theorem 2 Let $M^{4}$ be a compact PL 4-manifold with non-empty connected boundary and let $K$ be a contracted triangulation of $M^{4}$ with 4 as (unique) singular color.

(a) If $K$ contains exactly one edge between two pairs of non-singular vertices, involving three vertices, then $M^{4}$ admits a handle decomposition without 1-handles;

(b) if $K$ contains exactly one edge between three pairs of vertices, involving all five vertices, and so that only one pair involves color 4 , then $M^{4}$ admits a special handlebody decomposition.

According to a well-known literature (see, for example, [30] or [26]), a framed link (possibly with dotted circles) can be associated to any handle decomposition of a closed PL 4-manifold: roughly speaking, dotted circles represent 1-handles, while undotted components give the instructions about the attachment of 2-handles. Then, a celebrated result by Montesinos and Laudenbach-Poenaru (31] and [29]) ensures that there is a unique way to add the (possible) 3 -handles and the 4-handle, so to obtain the closed 4-manifold. For this reason, given a framed link with $r \geq 0$ dotted circles $(L, c)$, it is usual to denote by $M^{3}(L, c)$ the 3 -manifold obtained from $\#_{r}\left(\mathbb{S}^{2} \times \mathbb{S}^{1}\right)$ by Dehn surgery along the undotted components of $(L, c)$, by $M^{4}(L, c)$ the compact PL 4-manifold (with boundary $M^{3}(L, c)$ ) obtained from $\mathbb{D}^{4}$ by attaching 1 - and 2- handles according to the framed link $(L, c)$ and - in case

\footnotetext{
${ }^{1}$ Actually, the result holds in general dimension $n$, by simply substituting $n+1$ to 5 , and it is usually stated in terms of the associated graphs, by making use of the notion of crystallization, which gives the name to the whole combinatorial representation theory via edge-colored graphs: see [17], or Section 2 of the present paper.
} 
$M^{3}(L, c)$ is PL-homeomorphic to either $\mathbb{S}^{3}$ or a connected sum of $s \geq 0$ copies of the orientable $\mathbb{S}^{2}$ bundle over $\mathbb{S}^{1}$ - by $\overline{M^{4}(L, c)}$ the closed PL 4-manifold obtained from $M^{4}(L, c)$ by adding $s$ 3-handles and one 4-handle.

In the connected boundary case, under the assumption of simply-connectedness, Trace proved a uniqueness property for the attachment of 3-handles:

Proposition 3 [33, Theorem 1] Let $N_{1}^{4}, N_{2}^{4}$ be compact simply-connected PL 4-manifolds with nonempty connected boundary, so that $N_{i}^{4}$ is obtained from the compact 4-manifold $W_{i}^{4}(\forall i \in\{1,2\})$ by adding $s \geq 0$ 3-handles. Then, $N_{1}^{4}$ is PL-homeomorphic to $N_{2}^{4}$ if and only if $W_{1}^{4}$ is PL-homeomorphic to $W_{2}^{4}$.

As Trace himself points out, Proposition 3 implies "that the essential structure of such 4-manifolds is contained in a neighborhood of their 2-skeleton". Hence, framed links (possibly with dotted circles), together with the number of required 3-handles, can unambiguously identify compact simplyconnected PL 4-manifolds with non-empty connected boundary.

As a consequence, if $M^{4}(L, c)^{(s)}$ denotes the simply connected compact PL 4-manifold obtained from $M^{4}(L, c)$ by adding $s$-handles, then both Theorems 1 and 2 may be re-stated as follows:

\section{Proposition 4}

(a) If $M^{4}$ is a closed PL 4-manifold (resp. a compact PL 4-manifold with connected non-empty boundary) satisfying the hypothesis of Theorem 1 (a) (resp. Theorem $2(a)$ ), then a framed link $(L, c)$ (with no dotted circle) and an integer $s \geq 0$ exist, so that:

$$
\begin{gathered}
M^{3}(L, c)=\#{ }_{s}\left(\mathbb{S}^{2} \times \mathbb{S}^{1}\right) \quad \text { and } \quad \overline{M^{4}(L, c)}=M^{4} \\
\text { resp. } \left.\quad M^{3}(L, c)=\# s\left(\mathbb{S}^{2} \times \mathbb{S}^{1}\right) \# \partial M^{4} \quad \text { and } \quad M^{4}(L, c)^{(s)}=M^{4}\right) .
\end{gathered}
$$

(b) If $M^{4}$ is a closed PL 4-manifold (resp. a compact PL 4-manifold with connected non-empty boundary) satisfying the hypothesis of Theorem 1 (b) (resp. Theorem Q (b)), then a framed link $(L, c)$ (with no dotted circle) exists, so that:

$$
\begin{gathered}
M^{3}(L, c)=\mathbb{S}^{3} \quad \text { and } \quad \overline{M^{4}(L, c)}=M^{4} \\
\text { (resp. } \left.\quad M^{3}(L, c)=\partial M^{4} \quad \text { and } \quad M^{4}(L, c)=M^{4}\right) .
\end{gathered}
$$

Within crystallization theory, two interesting classes of graphs representing compact simplyconnected PL 4-manifolds (with empty or connected boundary) have been recently introduced and studied: they are called weak-simple and simple crystallizations and are proved to minimize combinatorially defined PL-invariants, i.e. the regular genus and, in case of simple crystallizations, also the gem-complexity and the G-degree (see [15] and references therein, or Section 3).

Since weak simple (resp. simple) crystallizations - by definition - combinatorially visualize contracted colored triangulations which admit exactly one edge between two vertices labelled by colors consecutive in a chosen permutation of the color set (resp. between any pair of vertices 2 ), the represented 4-manifold trivially satisfy the hypothesis of Theorem 1 (b) (in the closed case) or Theorem 2 (b) (in the connected boundary case). Hence, the following result is a direct consequence of Theorem 1 and Theorem 2, together with Proposition 4:

Theorem 5 Let $M^{4}$ be a compact PL 4-manifold with empty or connected boundary. If $M^{4}$ admits a weak simple crystallization, then $M^{4}$ admits a special handlebody decomposition (or, equivalently, $M^{4}$ is represented by a (not dotted) framed link with $\beta_{2}\left(M^{4}\right)$ components).

\footnotetext{
${ }^{2}$ Note that, as their names suggest, the class of simple crystallization is a subclass of that of weak simple ones; actually, they both are particular cases of even larger classes of crystallizations, representing not simply-connected compact PL 4manifolds, and still minimizing combinatorially defined PL invariants, i.e. weak semi-simple and semi-simple crystallizations. Precise definitions of all these classes may be found in [15, together with its references.
} 
Note that the above result extends to the connected boundary case a property already proved in [14 for closed PL 4-manifolds admitting simple crystallizations; moreover, both in the closed and in the boundary case, our statement takes into account also the larger class of weak simple crystallizations. This latter extension is of particular interest with respect to the quoted Kirby's problem, since it is not difficult to prove the existence of (closed) simply-connected PL 4-manifolds which can't admit simple crystallizations, while it is an open question the possible existence of weak simple crystallizations.

For this reason, we formulate the following

Question 1 Does any closed simply-connected PL 4-manifold admits a weak-simple crystallization?

In virtue of Theorem [5, a positive answer to the above question implies the universality of simple handlebody decompositions for any closed simply-connected 4-manifold; moreover, since 4-manifolds admitting weak simple crystallizations are proved to be characterized by the equality between their regular genus and twice their second Betti number (Proposition 10), it implies also the non-existence of exotic copies of $\mathbb{S}^{4}$ (and hence the 4-dimensional Poincaré conjecture in smooth category) and of $\mathbb{C P}^{2}$.

Moreover, via Proposition 10, it is not difficult to check the equivalence between Question 1 and a deeply investigated conjecture settled in 1986 within crystallization theory:

Conjecture 1 21] The graph-defined PL invariant regular genus is additive with respect to connected sum of closed simply-connected 4-manifolds.

Actually, the achievements of the present paper suggest to formulate the following weaker conjecture, still having all the described implications as regards exotic structures, Poincaré Conjecture and special handlebody decompositions:

Conjecture 2 Any closed simply-connected PL 4-manifold admits a contracted triangulation satisfying the hypothesis of Theorem 1 (b).

Finally, we point out that the described open questions might be faced also via a computational approach, in virtue of the totally combinatorial nature of the chosen representing tool: see for example [12], yielding an algorithmic procedure for generating and studying a 4-manifolds catalogue via colored graphs.

\section{Preliminaries}

In this section we will introduce the basic notions of the representation theory of compact PL manifolds by means of regular edge-colored graphs; further details can be found in [21]. All manifolds are supposed to be PL and, when not otherwise stated, connected.

Definition 1 A singular $n$-manifold is a closed connected $n$-dimensional polyhedron admitting a simplicial triangulation where the links of vertices are closed connected $(n-1)$-manifold 3 . Vertices whose links are not $(n-1)$-spheres are called singular.

Remark 1 If $N$ is a singular $n$-manifold, then deleting small open neighbourhoods of its singular vertices yields a compact $n$-manifold $\check{N}$ with no spherical boundary components. Conversely, from any compact $n$-manifold $M$, a singular $n$-manifold $\widehat{M}$ can be constructed by capping off each component of $\partial M$ by a cone over it.

Moreover, within the class of compact $n$-manifolds with no spherical boundary components, that we will consider throughout the present work, the above correspondence is well-defined, therefore singular $n$-manifolds and compact $n$-manifolds of this class can be bijectively associated.

\footnotetext{
${ }^{3}$ Note that, as a consequence, the links of all $h$-simplices of the triangulation with $h>0$ are $(n-h-1)$-spheres.
} 
Definition 2 Given a singular $n$-manifold $N$, a colored triangulation of $N$ is a pseudocomplex $K$ triangulating $N$ and carrying a vertex-labelling by means of the elements of the set $\Delta_{n}=\{0, \ldots, n\}$, such that vertices belonging to the same simplex have different labels.

Given an $h$-simplex $(0 \leq h \leq n) \sigma$ of $K$, the disjoint star of $\sigma$ in $K$ is the pseudocomplex obtained by taking the $n$-simplexes of $K$ which contain $\sigma$ and identifying, according to the gluings in $K$, only their $(n-1)$-faces containing $\sigma$. The disjoint link of $\sigma$ in $K$ is the subcomplex of its disjoint star formed by the simplexes that do not contain $\sigma$.

Definition 3 An $(n+1)$-colored graph $(n \geq 2)$ is a pair $(\Gamma, \gamma)$, where $\Gamma=(V(\Gamma), E(\Gamma))$ is an $(n+1)$-regular multigraph (i.e. multiple edges are allowed, but loops are forbidden) and $\gamma$ is an edge-coloration, that is a map $\gamma: E(\Gamma) \rightarrow \Delta_{n}=\{0, \ldots, n\}$ assigning different colors to incident edges.

Given $\left\{c_{1}, \ldots, c_{h}\right\} \subseteq \Delta_{n}$, let us set $\hat{c}_{1} \cdots \hat{c}_{h}=\Delta_{n} \backslash\left\{c_{1}, \ldots, c_{h}\right\}$; by $\Gamma_{c_{1}, \ldots, c_{h}}$ (resp. $\Gamma_{\hat{c}_{1} \ldots \hat{c}_{h}}$ ) we will denote the spanning subgraph of $(\Gamma, \gamma)$ whose edge set contains only the edges of $\Gamma$ that are colored (resp. that are not colored) by $\left\{c_{1}, \ldots, c_{h}\right\}$. The connected components of $\Gamma_{c_{1}, \ldots, c_{h}}$ will be called $\left\{c_{1}, \ldots, c_{h}\right\}$-residues of $\Gamma$ and their number will be denoted by $g_{c_{1}, \ldots, c_{h}}$. Furthermore, we will use the notation $g_{\hat{c}_{1} \cdots \hat{c}_{h}}$ for the number of connected components of $\Gamma_{\hat{c}_{1} \cdots \hat{c}_{h}}$.

Given a colored triangulation $K$ of a singular $n$-manifold $N$, the dual 1-skeleton of $K$ is an $(n+1)$ colored graph $(\Gamma(K), \gamma(K))$, whose edges inherit the coloration from the vertex-labelling of $K$; more precisely, for each $c \in \Delta_{n}$, an edge $e \in E(\Gamma(K))$ is $c$-colored iff the $(n-1)$-simplex of $K$ dual to $e$ misses color $c$.

We will say that $(\Gamma(K), \gamma(K))$ represents the singular manifold $N$ and, in virtue of Remark 1, also the compact $n$-manifold $N$.

The duality between $K$ and $(\Gamma(K), \gamma(K))$ gives rise to a bijective correspondence between $(n-h)$ simplexes of $K$ and $h$-residues of $\Gamma(K)$; more precisely, to each $(n-h)$-simplex $\sigma$ of $K$ whose vertices are labelled by $\hat{c}_{1} \cdots \hat{c}_{h}$ corresponds the $\left\{c_{1}, \ldots, c_{h}\right\}$-residue of $\Gamma(K)$ representing the disjoint link of $\sigma$ in $K$. Conversely, any $(n+1)$-colored graph $(\Gamma, \gamma)$ can be thought as the dual 1-skeleton of a pseudocomplex $K(\Gamma)$, whose $n$-simplexes are in bijection with the vertices of $\Gamma$ and the gluings of $(n-1)$-faces are determined by the colored adjacencies of $\Gamma$ (see [21] for a more detailed description of this construction).

By the duality between $(\Gamma, \gamma)$ and $K(\Gamma)$ it is not difficult to see that $|K(\Gamma)|$ is a singular $n$-manifold iff for any $c \in \Delta_{n}$, each $\hat{c}$-residue of $\Gamma$ represents a closed connected $(n-1)$-manifold5; residues not representing $(n-1)$-spheres are called singular. In particular, $|K(\Gamma)|$ is a closed $n$-manifold iff for any $c \in \Delta_{n}$, no $\hat{c}$-residue of $\Gamma$ is singular.

Throughout the paper we will be interested in colored graphs representing singular $n$-manifolds with at most one singular vertex, whose associated compact manifolds have therefore empty or connected boundary. In particular, we will consider $(n+1)$-colored graphs whose dual colored triangulations have the minimum possible number of vertices.

Definition 4 An $(n+1)$-colored graph $(\Gamma, \gamma)$ representing a compact $n$-manifold $M$ with empty or connected boundary, is called a crystallization of $M$ if $g_{\hat{c}}=1, \forall c \in \Delta_{n}$.

Its dual colored triangulation $K(\Gamma)$, having exactly $n+1$ vertices, is said to be contracted.

Remark 2 The existence of combinatorial moves (dipole moves) on edge-colored graphs inducing PL homeomorphisms between the represented compact manifolds, allows to prove that any compact n-manifold with empty or connected boundary admits a crystallization ([15]).

The possibility of defining, for compact manifolds, the PL invariant regular genus relies on the existence of a particular type of embedding of colored graphs into surfaces.

\footnotetext{
${ }^{4}$ The notion of pseudocomplex is a generalization of that of simplicial complex. Roughly speaking, in a pseudocomplex two $n$-simplexes may have more than one common $(n-1)$-face; however identifications of faces of the same simplex are not allowed.

${ }^{5}$ Note that, in this case, each $\left\{c_{1}, \ldots, c_{h}\right\}$-residue $(1 \leq h<n)$ of $\Gamma$ represents an $(n-h-1)$-sphere.
} 
Definition 5 A cellular embedding of an $(n+1)$-colored graph $(\Gamma, \gamma)$ into a closed surface is called regular if there exists a cyclic permutation $\varepsilon$ of $\Delta_{n}$ such that the regions of the embedding are bounded by the images of the cycles of $\Gamma$ whose edges are colored by two colors that are consecutive in $\varepsilon$.

An important result by Gagliardi ([22]) guarantees that given a bipartite (resp. non-bipartite) $(n+1)$-colored graph $(\Gamma, \gamma)$, for each cyclic permutation $\varepsilon=\left(\varepsilon_{0}, \ldots, \varepsilon_{n}\right)$ of $\Delta_{n}$ there exists a regular embedding of $(\Gamma, \gamma)$ such that the embedding surface $F_{\varepsilon}(\Gamma)$ is orientable (resp. non-orientable) and has genus (resp. half genus) $\rho_{\varepsilon}(\Gamma)$ satisfying

$$
2-2 \rho_{\varepsilon}(\Gamma)=\sum_{j \in \mathbb{Z}_{n+1}} g_{\varepsilon_{j}, \varepsilon_{j+1}}+(1-n) p,
$$

where $2 p$ is the order of $\Gamma$.

$\rho_{\varepsilon}(\Gamma)$ is called the regular genus of $\Gamma$ with respect to the permutation $\varepsilon$.

Moreover, $\varepsilon$ and $\varepsilon^{-1}$ induce the same embedding and $(\Gamma, \gamma)$ admits no regular embeddings into non-orientable (resp. orientable) surfaces.

As a consequence, the following definitions can be given:

Definition 6 The regular genus $\rho(\Gamma)$ of an $(n+1)$-colored graph $(\Gamma, \gamma)$ is defined as

$$
\rho(\Gamma)=\min \left\{\rho_{\varepsilon}(\Gamma) / \varepsilon \text { is a cyclic permutation of } \Delta_{n}\right\} .
$$

The (generalized) regular genus of a compact $n$-manifold $M(n \geq 2)$ is defined as

$$
\mathcal{G}(M)=\min \{\rho(\Gamma) \mid(\Gamma, \gamma) \text { represents } M\}
$$

Remark 3 Any bipartite (resp. non-bipartite) 3-colored graph $(\Gamma, \gamma)$ represents an orientable (resp. non-orientable) surface $|K(\Gamma)|$ and $\rho(\Gamma)$ coincides with the genus (resp. half the genus) of $|K(\Gamma)|$; moreover, the regular genus of a closed 3-manifold coincides with its Heegaard genus and the coincidence still holds in the case of connected boundary ([22, [19]) making the regular genus an effective extension to dimension $n>3$ of these classical notions of genus.

Actually many classification results according to this invariant already exist in dimension 4 and 5 ([7], [18, [13], 9] and references therein).

Presentations of the fundamental groups of a compact $n$-manifold and its associated singular manifold can be obtained from any $(n+1)$-colored graph representing them in the following way ([9]):

Proposition 6 Let $(\Gamma, \gamma)$ be an $(n+1)$-colored graph representing a compact $n$-manifold $M$.

For each $i, j \in \Delta_{n}$, let $X_{i j}$ (resp. $R_{i j}$ ) be a set in bijection with the connected components of $\Gamma_{\hat{i} \hat{j}}$ (resp. with the $\{i, j\}$-colored cycles of $\Gamma)$, and let $\bar{R}_{i j}$ be a subset of $X_{i j}$ corresponding to the a maximal tree of the subcomplex $K_{i j}$ of $K(\Gamma)$ (consisting only of vertices labelled $i$ and $j$, and edges connecting them). Then:

(a) if $i, j \in \Delta_{n}$ are not singular in $\Gamma$,

$$
\pi_{1}(M)=<X_{i j} / R_{i j} \cup \bar{R}_{i j}>\text {; }
$$

(a') if no color in $\Delta_{n}-\{i, j\}$ is singular in $\Gamma$,

$$
\pi_{1}(\widehat{M})=<X_{i j} / R_{i j} \cup \bar{R}_{i j}>.
$$




\section{Weak simple crystallizations of compact 4-manifolds}

In [5] and [3] semi-simple and weak semi-simple crystallizations of closed 4-manifolds are introduced and studied, by generalizing the former notion of simple crystallizations of closed simply-connected 4-manifolds (see [4] and [14]): they are proved to be "minimal" with respect to regular genus, among all graphs representing the same closed 4-manifold. In [11] these definitions are extended to compact 4-manifolds with empty or connected boundary and their minimizing property with respect to regular genus is proved to hold also in this wider context ([15]) 6

Since the present paper focuses on simply-connected 4-manifolds (with empty or connected boundary), we will restrict the definitions to this particular case, where the above types of crystallizations are called simple and weak simple respectively.

In the following, given a 5-colored graph $(\Gamma, \gamma)$ representing a compact 4-manifold with empty or connected boundary, we will always assume, without loss of generality, that color 4 is its (unique) possible singular color.

Furthermore, $\mathcal{P}_{4}$ will denote the set of all cyclic permutations of $\Delta_{4}$, and, given $\varepsilon=\left(\varepsilon_{0}, \varepsilon_{1}, \varepsilon_{2}, \varepsilon_{3}, \varepsilon_{4}\right) \in$ $\mathcal{P}_{4}$, we will always suppose, again without loss of generality, $\varepsilon_{4}=4$.

We can now introduce the following definitions:

Definition 7 Let $M^{4}$ be a compact simply-connected 4-manifold, with empty or connected boundary. A 5-colored graph $(\Gamma, \gamma)$ representing $M^{4}$ is called weak simple with respect to $\varepsilon \in \mathcal{P}_{4}$ if $g_{\varepsilon_{i}, \varepsilon_{i+2}, \varepsilon_{i+4}}=$ $1 \forall i \in \Delta_{4}$ (where the additions in subscripts are intended in $\mathbb{Z}_{5}$ ). $(\Gamma, \gamma)$ is called simple if $g_{j, k, l}=1 \quad \forall j, k, l \in \Delta_{4}$.

Note that, as a consequence of the above definition, if $(\Gamma, \gamma)$ is weak simple, then $g_{\hat{j}}=1, \forall j \in \Delta_{4}$, i.e. $(\Gamma, \gamma)$ is a crystallization of $M^{4}$.

Let $(\Gamma, \gamma)$ be a crystallization of a compact simply-connected 4-manifold $M^{4}$ with empty or connected boundary (i.e. $\Gamma_{\hat{c}}$ represents $\mathbb{S}^{3}$, for any $c \neq 4$ ), and let us denote, for shortness, by $\rho_{\varepsilon}$ (resp. by $\rho_{\varepsilon_{\hat{i}}}$ ) the regular genus of $\Gamma$ (resp. of $\Gamma_{\widehat{\varepsilon_{i}}}$ ) with respect to the permutation $\varepsilon$ (resp. $\left.\varepsilon_{\hat{i}}=\left(\varepsilon_{0}, \ldots, \varepsilon_{i-1}, \varepsilon_{i+1}, \ldots, \varepsilon_{4}=4\right)\right)$.

By setting $g_{j, k, l}=1+t_{j, k, l} \quad j, k, l \in \Delta_{4}$ (see [3] and [1]), the following equality can be proved (see [11] and [15]):

$$
\rho_{\varepsilon}-\rho_{\varepsilon_{\hat{i}}}-\rho_{\varepsilon_{\overline{i+2}}}=t_{\varepsilon_{i-1}, \varepsilon_{i+1}, \varepsilon_{i+3}} \geq 0 \quad \forall i \in \Delta_{4}
$$

where all subscripts are taken in $\mathbb{Z}_{5}$.

By making use of relation (11), it is not difficult to prove the following characterization of weak simple crystallizations:

Proposition 7 ([11, Corollary 8]) Let $(\Gamma, \gamma)$ be a crystallization of a compact simply-connected 4manifold $M^{4}$ with empty or connected boundary. Then $(\Gamma, \gamma)$ is weak simple with respect to $\varepsilon \in \mathcal{P}_{4}$ if and only if for each $i \in \Delta_{4}, \rho_{\varepsilon_{\hat{i}}}=\frac{1}{2} \rho_{\varepsilon}$.

The Main Theorem of [15] explains the minimizing property of weak semi-simple crystallizations with respect to regular genus. In the simply-connected case, the result becomes:

\footnotetext{
${ }^{6}$ Actually, weak semi-simple crystallizations turn out to admit also interesting properties with respect to the existence of trisections of the represented compact 4-manifolds, and to the computation of their trisection genus: see [11] and references therein.
} 
Theorem 8 [15, Main Theorem] Let $M^{4}$ be a compact simply-connected 4-manifold with empty or connected boundary, then the regular genus $\mathcal{G}\left(M^{4}\right)$ of $M^{4}$ satisfies

$$
\mathcal{G}\left(M^{4}\right) \geq 2 \chi\left(\widehat{M}^{4}\right)-4 .
$$

Moreover, equality holds if and only if $M^{4}$ admits a weak simple crystallization.

Remark 4 We point out that simple, or semi-simple in the not simply-connected case, crystallizations realize also the PL invariants gem-complexity and G-degree ([15]). Gem-complexity of a compact PL 4-manifold $M^{4}$ is related to the minimum number of vertices in a 5-colored graph representing $M^{4}$, while the G-degree is a PL invariant arising within the theory of Colored Tensor Models, that was first developed, in theoretical physics, as a random geometry approach to quantum gravity and has subsequently gained interest in the study of quantum mechanical models, too (see [23, [16], 24], [34]).

As a consequence of Theorem 8, together with Proposition 17, we have:

Corollary 9 Let $(\Gamma, \gamma)$ be a crystallization of a compact simply-connected 4-manifold $M^{4}$ with empty or connected boundary, then, $(\Gamma, \gamma)$ is weak simple with respect to $\varepsilon \in \mathcal{P}_{4}$ if and only if

$$
\mathcal{G}\left(M^{4}\right)=\rho(\Gamma)=\rho_{\varepsilon}(\Gamma)=2 \chi\left(\widehat{M}^{4}\right)-4
$$

or, equivalently, if and only if $\rho_{\varepsilon_{\hat{\imath}}}=\chi\left(\widehat{M}^{4}\right)-2 \quad \forall i \in \Delta_{4}$.

Proposition 10 If $M^{4}$ is a simply-connected 4-manifold with empty or connected boundary, then

$$
\mathcal{G}\left(M^{4}\right) \geq 2 \beta_{2}\left(M^{4}\right)
$$

where $\beta_{2}\left(M^{4}\right)$ is the second Betti number of $M^{4}$.

Moreover, $\mathcal{G}\left(M^{4}\right)=2 \beta_{2}\left(M^{4}\right)$ holds if and only if $M^{4}$ admits a weak simple crystallization $(\Gamma, \gamma)$ with respect to $\varepsilon$ of $\mathcal{P}_{4}$ or, equivalently, if and only if all $\hat{\varepsilon}_{i}$-residues ( $i \in \mathbb{Z}_{5}$ ) of $\Gamma$ have regular genus $\beta_{2}\left(M^{4}\right)$ with respect to the permutations induced by $\varepsilon$.

Proof. First, let us observe that, for any compact 4-manifold $M^{4}$, if $C$ is the cone over $\partial M^{4}$, then the inclusion $(M, \partial M) \hookrightarrow\left(\widehat{M}^{4}, C\right)$ induces an isomorphism in homology; therefore the exact sequence of relative homology of the pair $\left(\widehat{M}^{4}, C\right)$, yields

$$
H_{k}\left(\widehat{M}^{4}\right) \cong H_{k}\left(\widehat{M}^{4}, C\right) \cong H_{k}\left(M^{4}, \partial M^{4}\right) \cong H^{4-k}\left(M^{4}\right) \quad \forall k \in\{2,3\}
$$

where the last isomorphism comes from Poincaré-Lefschetz duality.

Hence $\beta_{2}\left(\widehat{M}^{4}\right)=\beta_{2}\left(M^{4}\right), \beta_{3}\left(\widehat{M}^{4}\right)=\beta_{1}\left(M^{4}\right)$ and, therefore

$$
\chi\left(\widehat{M}^{4}\right)=2-\beta_{1}\left(\widehat{M}^{4}\right)+\beta_{2}\left(M^{4}\right)-\beta_{1}\left(M^{4}\right) .
$$

By further assuming $M^{4}$ to be simply-connected with empty or connected boundary, statement (a) of the Main Theorem implies

$$
\mathcal{G}\left(M^{4}\right) \geq 2 \chi\left(\widehat{M}^{4}\right)-4=2 \beta_{2}\left(M^{4}\right) .
$$

Now, by Corollary 9, a crystallization $\Gamma$ of $M^{4}$ realizes the lower bound for regular genus, i.e. $\rho(\Gamma)=2 \beta_{2}\left(M^{4}\right)$, if and only if there exists a cyclic permutation $\varepsilon$ of $\mathcal{P}_{4}$ such that $\Gamma$ is weak simple with respect to $\varepsilon$ (in this case $\rho(\Gamma)=\rho_{\varepsilon}(\Gamma)$ ).

Finally, Corollary 9 ensures the equivalence with the condition

$$
\rho_{\varepsilon_{\hat{\imath}}}=\chi\left(\widehat{M}^{4}\right)-2=\beta_{2}\left(M^{4}\right) \quad \forall i \in \Delta_{4} .
$$




\section{Proof of the main results}

In order to prove the main results of the paper, we need two lemmas.

Lemma 11 Let $M^{4}$ be a compact simply-connected 4-manifold. Then, for each 5-colored graph $(\Gamma, \gamma)$ representing $M^{4}$ and for each cyclic permutation $\varepsilon$ of $\Delta_{4}$,

$$
\sum_{i \in \Delta_{4}} \rho_{\varepsilon_{\hat{\imath}}}\left(\Gamma_{\widehat{\varepsilon_{i}}}\right)-2 \rho_{\varepsilon}(\Gamma)=\beta_{2}\left(M^{4}\right)
$$

As a consequence,

$$
\beta_{2}\left(M^{4}\right) \leq \min \left\{\rho_{\varepsilon_{\hat{i}}}\left(\Gamma_{\widehat{\varepsilon}_{i}}\right) /(\Gamma, \gamma) \text { representing } M^{4}, \varepsilon \text { cyclic permutation of } \Delta_{4}, i \in \Delta_{4}\right\} .
$$

Proof. According to formula (20) of Proposition 13 in [9], for each 5-colored graph representing a compact 4-manifold $M^{4}$ and for each cyclic permutation $\varepsilon$ of $\Delta_{4}$, the following relation holds:

$$
\chi\left(\widehat{M}^{4}\right)=2-2 \rho_{\varepsilon}+\sum_{i \in \Delta_{4}} \rho_{\varepsilon_{\hat{i}}} .
$$

By comparing with formula (2), we obtain:

$$
\sum_{i \in \Delta_{4}} \rho_{\varepsilon_{\hat{i}}}-2 \rho_{\varepsilon}=\beta_{2}\left(M^{4}\right)-\beta_{1}\left(M^{4}\right)-\beta_{1}\left(\widehat{M}^{4}\right)
$$

If $M^{4}$ is assumed to be simply-connected, the general relation of the statement directly follows.

On the other hand, it is well known that, for each 5-colored graph representing a compact 4manifold $M^{4}$ and for each $\varepsilon=\left(\varepsilon_{0}, \varepsilon_{1}, \varepsilon_{2}, \varepsilon_{3}, \varepsilon_{4}\right) \in \mathcal{P}_{4}$, the inequality

$$
\rho_{\varepsilon_{\widehat{j-1}}}+\rho_{\widehat{\varepsilon_{\widehat{j+1}}}} \leq \rho_{\varepsilon}
$$

holds $\forall j \in \Delta_{4}$ : see formula (24) of Proposition 7.1 in [9].

Hence, the hypothesis of simply-connectedness implies, $\forall i \in \Delta_{4}$ :

$$
\beta_{2}\left(M^{4}\right)=\sum_{i \in \Delta_{4}} \rho_{\varepsilon_{\hat{i}}}-2 \rho_{\varepsilon}=\rho_{\varepsilon_{\hat{i}}}+\left(\rho_{\varepsilon_{\widehat{i}-1}}+\rho_{\varepsilon_{\widehat{i+1}}}\right)+\left(\rho_{\varepsilon_{\widehat{i-2}}}+\rho_{\varepsilon_{\overparen{i+1}}}\right)-2 \rho_{\varepsilon} \leq \rho_{\varepsilon_{\hat{i}}} .
$$

Lemma 12 Let $(\Gamma, \gamma)$ be a crystallization of a compact 4-manifold $M^{4}$ (with empty or connected boundary), such that color 4 is the only possible singular color. If there exist two different colors $j, k \in \Delta_{3}$ such that $g_{\hat{j} \hat{k}}=1$, then $M^{4}$ is simply-connected and for each $s \in \Delta_{3}-\{j, k\}$, a cyclic permutation $\varepsilon$ of $\Delta_{4}$ exists, with $\varepsilon_{0}=s$, so that:

$$
\rho_{\varepsilon_{\hat{0}}}=\beta_{2}\left(M^{4}\right)+t_{s, j, k} .
$$

In particular, if there exist three different colors $r, j, k \in \Delta_{3}$ such that $g_{\hat{r} \hat{u}}=g_{\hat{j} \hat{k}}=1$, then a cyclic permutation $\varepsilon$ of $\Delta_{4}$ exists, so that $\rho_{\varepsilon_{\hat{0}}}=\beta_{2}\left(M^{4}\right)$, where $\left\{\varepsilon_{0}\right\}=\Delta_{4}-\{r, j, k, 4\}$.

Proof. According to Section 4 of [11], for each crystallization of a compact 4-manifold with either empty or connected boundary, setting $m=r k\left(\pi_{1}\left(M^{4}\right)\right)$ and $m^{\prime}=r k\left(\pi_{1}\left(\widehat{M^{4}}\right)\right)$, we have:

$$
\begin{array}{cc}
g_{\varepsilon_{i-1}, \varepsilon_{i+1}, \varepsilon_{i+3}}=1+\rho_{\varepsilon}-\rho_{\varepsilon_{\hat{i}}}-\rho_{\varepsilon_{\overline{i+2}}}=1+m^{\prime}+t_{\varepsilon_{i-1}, \varepsilon_{i+1}, \varepsilon_{i+3}} & \forall i \in\{2,4\} \text { and } \\
g_{\varepsilon_{i-1}, \varepsilon_{i+1}, \varepsilon_{i+3}}=1+\rho_{\varepsilon}-\rho_{\varepsilon_{\hat{\imath}}}-\rho_{\varepsilon_{\widehat{i+2}}}=1+m+t_{\varepsilon_{i-1}, \varepsilon_{i+1}, \varepsilon_{i+3}} & \forall i \in\{0,1,3\}
\end{array}
$$


which trivially imply

$$
\begin{aligned}
\rho_{\varepsilon}-\rho_{\varepsilon_{\hat{i}}}-\rho_{\varepsilon_{\overparen{i+2}}}-m^{\prime}=t_{\varepsilon_{i-1}, \varepsilon_{i+1}, \varepsilon_{i+3}} & \forall i \in\{2,4\} \text { and } \\
\rho_{\varepsilon}-\rho_{\varepsilon_{\hat{\imath}}}-\rho_{\varepsilon_{\overparen{i+2}}}-m=t_{\varepsilon_{i-1}, \varepsilon_{i+1}, \varepsilon_{i+3}} & \forall i \in\{0,1,3\} .
\end{aligned}
$$

Let $\varepsilon$ be a cyclic permutation of $\Delta_{4}$ with $\varepsilon_{0}=s$ and such that both $r, 4$ and $j, k$ are not consecutive, $r$ being the only element of $\Delta_{2}-\{s, j, k\}$; for example, let us suppose : $\varepsilon=\left(\varepsilon_{0}=s, \varepsilon_{1}=j, \varepsilon_{2}=\right.$ $\left.r, \varepsilon_{3}=k, \varepsilon_{4}=4\right)$ ), then $g_{\hat{j} \hat{k}}=1$ implies $m=0$ (see Proposition 6 (a)), $t_{s, r, 4}=0$ and $\rho_{\varepsilon_{\hat{1}}}+\rho_{\varepsilon_{\hat{3}}}=\rho_{\varepsilon}$. On the other hand, $m=0$ implies $m^{\prime}=0$, and hence $\rho_{\varepsilon_{\hat{2}}}+\rho_{\varepsilon_{\hat{4}}}=\rho_{\varepsilon}-t_{s, j, k}$ As a consequence, $M^{4}$ is simply-connected and Lemma 11 easily yields $\rho_{\varepsilon_{\hat{0}}}-t_{s, j, k}=\beta_{2}\left(M^{4}\right)$.

The second part of the statement directly follows from the first one, by recalling that $g_{\hat{r} \hat{4}}=1$ means $t_{s, j, k}=0$.

Let us recall now that that every closed (resp. compact) 4-manifold $M^{4}$ admits a handle decomposition

$$
M^{4}=H^{(0)} \cup\left(H_{1}^{(1)} \cup \cdots \cup H_{r_{1}}^{(1)}\right) \cup\left(H_{1}^{(2)} \cup \cdots \cup H_{r_{2}}^{(2)}\right) \cup\left(H_{1}^{(3)} \cup \cdots \cup H_{r_{3}}^{(3)}\right) \cup H^{(4)}
$$

(resp.

$$
\left.M^{4}=H^{(0)} \cup\left(H_{1}^{(1)} \cup \cdots \cup H_{r_{1}}^{(1)}\right) \cup\left(H_{1}^{(2)} \cup \cdots \cup H_{r_{2}}^{(2)}\right) \cup\left(H_{1}^{(3)} \cup \cdots \cup H_{r_{3}}^{(3)}\right)\right)
$$

where $H^{(0)}=\mathbb{D}^{4}$ and each $p$-handle $H_{i}^{(p)}=\mathbb{D}^{p} \times \mathbb{D}^{4-p}\left(1 \leq p \leq 4,1 \leq i \leq r_{p}\right)$ is endowed with an embedding (called attaching map) $f_{i}^{(p)}: \partial \mathbb{D}^{p} \times \mathbb{D}^{4-p} \rightarrow \partial\left(H^{(0)} \cup \ldots\left(H_{1}^{(p-1)} \cup \cdots \cup H_{r_{p-1}}^{(p-1)}\right)\right.$ ); moreover, in the closed case, it is well-known that 3 - and 4-handles are attached in a unique way to the union of the $h$-handles, with $0 \leq h \leq 2$.

A standard argument of crystallization theory allows to state that, for any crystallization $(\Gamma, \gamma)$ of a closed 4-manifold $M^{4}$ and for any partition $\{\{i, j, k\},\{r, s\}\}$ of $\Delta_{4}, M^{4}$ admits a decomposition of type $M^{4}=N(i, j, k) \cup_{\phi} N(r, s)$, where $N(i, j, k)$ (resp. $\left.N(r, s)\right)$ denotes a regular neighbourhood of the subcomplex $K(i, j, k)$ (resp. $K(r, s)$ ) of $K(\Gamma)$ generated by the vertices labelled $\{i, j, k\}$ (resp. $\{r, s\})$ and $\phi$ is a boundary identification (see [20] and [22]). The same decomposition applies to the singular manifold $\widehat{M}^{4}$, in case $(\Gamma, \gamma)$ is a crystallization of a compact 4-manifold.

Any such decomposition turns out to induce a handle decomposition of the closed (resp. compact, with non-empty boundary) 4-manifold $M^{4}$, where $N(i, j, k)$ constitutes the union of the $h$-handles, with $0 \leq h \leq 2$, while $N(r, s)$ is the union of 3- and 4-handles (resp. is the union of 3-handles together with the cones over the components of $\left.\partial M^{4}\right)$.

The following proposition establishes combinatorial conditions on $(\Gamma, \gamma)$ which ensure the existence of particular types of handle decompositions of the represented manifold (lacking in 1-handles).

Proposition 13 Let $(\Gamma, \gamma)$ be a crystallization of a (simply-connected) compact 4-manifold $M^{4}$ (with empty or connected boundary), such that color 4 is the only possible singular color. If there exist three different colors $i, j, k \in \Delta_{3}$ such that $g_{\hat{i} \hat{k}}=g_{\hat{j} \hat{k}}=1$, then $M^{4}$ admits a handle decomposition consisting of one 0-handle, $\beta_{2}\left(M^{4}\right)+t_{i, j, k}$ 2-handles, $t_{i, j, k}$ 3-handles and - in the closed case - one 4-handle.

In particular, if there exists a partition $\{\{4, r\},\{i, j, k\}\}$ of $\Delta_{4}$ such that $g_{\hat{r} \hat{4}}=g_{\hat{i} \hat{k}}=g_{\hat{j} \hat{k}}=1$, then the induced handle decomposition of $M^{4}$ consists of one 0-handle, $\beta_{2}\left(M^{4}\right)$ 2-handles and-in the closed case - one 4-handle.

Proof. Let us denote by $\varepsilon$ the following cyclic permutation of $\Delta_{4}$ : $\varepsilon=\left(\varepsilon_{0}=i, \varepsilon_{1}=j, \varepsilon_{2}=r, \varepsilon_{3}=\right.$ $\left.k, \varepsilon_{4}=4\right)$, where $r$ is the only element of $\Delta_{3}-\{i, j, k\}$. Then, let us take into account the partition $\left\{\left\{\varepsilon_{0}, \varepsilon_{1}, \varepsilon_{3}\right\},\left\{\varepsilon_{2}, \varepsilon_{4}\right\}\right\}$ of $\Delta_{4}$ (i.e. $\left.\{\{4, r\},\{i, j, k\}\}\right)$. Now, in order to analyze the associated handle decomposition, first of all note that $K\left(\varepsilon_{2}, \varepsilon_{4}\right)=K(r, 4)$ consists of exactly $g_{\hat{r} \hat{u}}=1+t_{i, j, k} 1$-simplices; hence, $N\left(\varepsilon_{2}, \varepsilon_{4}\right)=N(r, 4)$ turns out to be PL-homeomorphic to the boundary connected sum between 
a handlebody $\mathbb{Y}_{t_{i, j, k}}^{4}$ and the cone $v_{4} * l k d\left(v_{4}, K(\Gamma)\right.$ ) (which is a cone over $\partial M^{4}$ in the connected boundary case, and a 4 -disk in the closed case), where $l k d$ denotes the disjoint link.

On the other hand, the assumption also implies that both $K\left(\varepsilon_{0}, \varepsilon_{3}\right)=K(i, k)$ and $K\left(\varepsilon_{1}, \varepsilon_{3}\right)=$ $K(j, k)$ consists of exactly one 1 -simplex, while $K\left(\varepsilon_{0}, \varepsilon_{1}\right)=K(i, j)$ consists of $g_{\varepsilon_{2}, \varepsilon_{3}, \varepsilon_{4}}=g_{\hat{i}, \hat{j}} \geq 1$ 1-simplices.

Moreover, by [9, Prop. 7.1] (and, in particular, by [9, formula (22)]):

$$
\begin{aligned}
g_{\widehat{\varepsilon_{0}}, \widehat{\varepsilon_{1}}, \widehat{\varepsilon_{3}}} & =g_{\varepsilon_{2}, \varepsilon_{4}}=\left(g_{\widehat{\varepsilon_{0}}, \widehat{\varepsilon_{1}}}+g_{\widehat{\varepsilon_{0}}, \widehat{\varepsilon_{3}}}-g_{\widehat{\varepsilon_{0}}}\right)+\rho_{\varepsilon_{\hat{0}}} \\
& =\left(g_{\varepsilon_{2}, \varepsilon_{3}, \varepsilon_{4}}+1-1\right)+\rho_{\varepsilon_{\hat{0}}}
\end{aligned}
$$

This means that $K\left(\varepsilon_{0}, \varepsilon_{1}, \varepsilon_{3}\right)$ contains $\rho_{\varepsilon_{0}}$ triangles more than edges in $K\left(\varepsilon_{0}, \varepsilon_{1}\right)$. Since each edge in $K\left(\varepsilon_{0}, \varepsilon_{1}\right)$ must be face of at least a triangle in $K\left(\varepsilon_{0}, \varepsilon_{1}, \varepsilon_{3}\right)$, then some triangles in $K\left(\varepsilon_{0}, \varepsilon_{1}, \varepsilon_{3}\right)$ may possibly collapse from their "free" face in $K\left(\varepsilon_{0}, \varepsilon_{1}\right)$, giving rise to a 2-dimensional complex $K^{\prime}\left(\varepsilon_{0}, \varepsilon_{1}, \varepsilon_{3}\right)$ consisting of $\rho_{\varepsilon_{0}}+h$ triangles, one edge in $K^{\prime}\left(\varepsilon_{0}, \varepsilon_{3}\right)=K\left(\varepsilon_{0}, \varepsilon_{3}\right)$, one edge in $K^{\prime}\left(\varepsilon_{1}, \varepsilon_{3}\right)=$ $K\left(\varepsilon_{1}, \varepsilon_{3}\right)$ and $h\left(1 \leq h \leq g_{\varepsilon_{2}, \varepsilon_{3}, \varepsilon_{4}}\right)$ edges in $K^{\prime}\left(\varepsilon_{0}, \varepsilon_{1}\right)$, such that, for each $i \in\{1, \ldots, h\}$, exactly $r_{i}$ triangles share the same edge in $K^{\prime}\left(\varepsilon_{0}, \varepsilon_{1}\right)$ (and hence have the same boundary), with $\sum_{i=1}^{h} r_{i}=\rho_{\varepsilon_{0}}+h$.

Now, it is not difficult to check that, if a "small" regular neighbourhood of the $\varepsilon_{0}$-labelled 0 -simplex of $K^{\prime}\left(\varepsilon_{0}, \varepsilon_{1}, \varepsilon_{3}\right)$, together with $h$ (arbitrarily fixed) triangles of $K^{\prime}\left(\varepsilon_{0}, \varepsilon_{1}, \varepsilon_{3}\right)$ having different faces in $K^{\prime}\left(\varepsilon_{0}, \varepsilon_{1}\right)$, is considered as a 0 -handle $H^{(0)}=\mathbb{D}^{4}$, then the regular neighbourhoods of the remaining $\rho_{\varepsilon_{0}}$ triangles in $K^{\prime}\left(\varepsilon_{0}, \varepsilon_{1}, \varepsilon_{3}\right)$ may be considered - by making use of Lemma 12- as $\rho_{\varepsilon_{\hat{0}}}=\beta_{2}\left(M^{4}\right)+t_{i, j, k}$ 2-handles attached on its boundary.

Hence, $N\left(\varepsilon_{0}, \varepsilon_{1}, \varepsilon_{3}\right)=H^{(0)} \cup\left(H_{1}^{(2)} \cup \cdots \cup H_{\beta_{2}\left(M^{4}\right)+t_{i, j, k}}^{(2)}\right)$, with $\partial\left(N\left(\varepsilon_{0}, \varepsilon_{1}, \varepsilon_{3}\right)\right)=\partial\left(N\left(\varepsilon_{2}, \varepsilon_{4}\right)\right)$ PL-homeomorphic to the connected sum between $\partial\left(\mathbb{Y}_{t_{i, j, k}}^{4}\right)=\#_{t_{i, j, k}}\left(\mathbb{S}^{2} \times \mathbb{S}^{1}\right)$ and $\partial M^{4}$ (in case of non-empty connected boundary).

The proof of the general statement is completed by noting that - in virtue of the important result of [31] and [29], extended in [33] to the case of simply connected 4-manifolds with connected boundary, as already recalled in Proposition 3 - the boundary identification $\phi$ between $N\left(\varepsilon_{0}, \varepsilon_{1}, \varepsilon_{3}\right)$ and $N\left(\varepsilon_{2}, \varepsilon_{4}\right)$ is nothing but the attachment of $t_{i, j, k} 3$-handles and, in the closed case, a 4-handle:

$$
\begin{aligned}
M^{4}=N\left(\varepsilon_{0}, \varepsilon_{1}, \varepsilon_{3}\right) \cup_{\phi} N\left(\varepsilon_{2}, \varepsilon_{4}\right) & =\left[H^{(0)} \cup\left(H_{1}^{(2)} \cup \cdots \cup H_{\beta_{2}\left(M^{4}\right)+t_{i, j, k}}^{(2)}\right)\right] \cup_{\phi} \mathbb{Y}_{t_{i, j, k}}^{4}= \\
& =H^{(0)} \cup\left(H_{1}^{(2)} \cup \cdots \cup H_{\beta_{2}\left(M^{4}\right)+t_{i, j, k}}^{(2)}\right) \cup\left(H_{1}^{(3)} \cup \cdots \cup H_{t_{i, j, k}}^{(3)}\right) \cup H^{(4)} .
\end{aligned}
$$

The second part of the statement directly follows form the first one, since $g_{\hat{r} \hat{4}}=1$ means $t_{i, j, k}=0$.

Remark 5 As already pointed out in the introduction, Proposition 13 can be equivalently formulated in terms of a relationship between crystallization theory and framed link representation of compact 4manifolds. The same relationship, in the inverse direction, is investigated in [10] (and - via a slightly different tool - in [6] and [8], too), by describing how to construct a 5-colored graph representing $\bar{M}^{4}(L, c)$ directly from $(L, c)$.

In virtue of the bijection between colored graphs and colored triangulations described in Section 2. both Theorem 1 and Theorem 2 directly follow from Proposition 13, while the proof of Theorem 5 is an easy consequence:

Proof of Theorem 5 . Let $(\Gamma, \gamma)$ be a weak simple crystallization of the simply-connected compact 4-manifold $M^{4}$ (with empty or connected boundary), with respect to the cyclic permutation $\varepsilon=\left(\varepsilon_{0}, \varepsilon_{1}, \varepsilon_{2}, \varepsilon_{3}, \varepsilon_{4}=4\right)$ of $\Delta_{4}$. By hypothesis, surely $g_{\hat{\varepsilon_{2}} \hat{4}}=g_{\hat{\varepsilon_{0}} \hat{\varepsilon_{3}}}=g_{\hat{\varepsilon_{1}} \hat{\varepsilon_{3}}}=1$. Hence, the second statement of Proposition 13 ensures that the partition $\left\{\left\{\varepsilon_{0}, \varepsilon_{1}, \varepsilon_{3}\right\},\left\{\varepsilon_{2}, \varepsilon_{4}\right\}\right\}$ of $\Delta_{4}$ induces a handle decomposition of $M^{4}$ consisting of one 0-handle, $\beta_{2}\left(M^{4}\right)$ 2-handles and - in the closed case - one 4-handle. This proves that $M^{4}$ is represented by a (not dotted) framed link with $\beta_{2}(M)$ components. 


\section{References}

[1] S. Akbulut, The Dolgachev Surface - Disproving Harer-Kas-Kirby Conjecture Comm. Math. Helvetici 87(1) (2012), 187-241.

[2] S. Akbulut, An infinite family of exotic Dolgachev surfaces without 1- and 3- handles, Jour. of GGT 3 (2009), 22-43.

[3] B.Basak, Genus-minimal crystallizations of PL 4-manifolds, Beitr. Algebra Geom. 59 (1) (2018), 101-111.

[4] B. Basak - J. Spreer, Simple crystallizations of 4-manifolds, Adv. Geom. 16 (1) (2016), 111-130.

[5] B. Basak - M.R. Casali, Lower bounds for regular genus and gem-complexity of PL 4-manifolds, Forum Math. 29 (4) (2017), 761-773.

[6] M.R. Casali, From framed links to crystallizations of bounded 4-manifolds, Journal of Knot Theory and its Ramifications 9 (4) (2000), 443-458.

[7] M.R. Casali, On the regular genus of 5-manifolds with free fundamental group, Forum Math. 15 (2003), 465-475.

[8] M. R. Casali, Dotted links, Heegaard diagrams and coloured graphs for PL 4-manifolds, Rev. Mat. Complut. 17(2) (2004) 435-457.

[9] M.R. Casali - P. Cristofori, Classifying compact 4-manifolds via generalized regular genus and G-degree, Ann. Inst. Henri Poincar D (2020), to appear. arXiv:1912.01302

[10] M.R. Casali - P. Cristofori, Kirby diagrams and 5-colored graphs representing compact 4manifolds, preprint 2020.

[11] M.R. Casali - P. Cristofori, Gem-induced trisections of compact PL 4-manifolds, preprint 2019. arXiv:1910.08777

[12] M.R. Casali - P. Cristofori, Cataloguing PL 4-manifolds by gem-complexity, The Electronic Journal of Combinatorics 22 (4) (2015), \#P4.25.

[13] M.R. Casali - P. Cristofori - C. Gagliardi, Classifying PL 4-manifolds via crystallizations: results and open problems, in: "Mathematical Tribute to Professor José María Montesinos Amilibia", Universidad Complutense Madrid (2016). [ISBN: 978-84-608-1684-3]

[14] M.R. Casali - P. Cristofori - C. Gagliardi, PL 4-manifolds admitting simple crystallizations: framed links and regular genus, Journal of Knot Theory and its Ramifications 25 (1) (2016), 1650005 [14 pages].

[15] M.R. Casali - P. Cristofori - C. Gagliardi, Crystallizations of compact 4-manifolds minimizing combinatorially defined PL-invariants, Rend. Istit. Mat. Univ. Trieste 52 (2020), to appear. arXiv:2004.07894

[16] M.R. Casali - P. Cristofori - S. Dartois - L. Grasselli, Topology in colored tensor models via crystallization theory, J. Geom. Phys. 129 (2018), 142-167.

[17] M.R. Casali - P. Cristofori - L. Grasselli, G-degree for singular manifolds, RACSAM 112 (3) (2018), 693-704.

[18] M.R.Casali - C.Gagliardi, Classifying PL 5-manifolds up to regular genus seven, Proc. Amer. Math. Soc. 120 (1994), 275-283.

[19] P. Cristofori - C.Gagliardi - L.Grasselli, Heegaard and regular genus of 3-manifolds with boundary, Rev. Mat. Univ. Complut. Madrid 8, (1995), 379-398. 
[20] M. Ferri - C. Gagliardi, The only genus zero n-manifold is $\mathbb{S}^{n}$, Proc. Amer. Math. Soc. 85 (1982), 638-642.

[21] M. Ferri - C. Gagliardi - L. Grasselli, A graph-theoretical representation of PL-manifolds. A survey on crystallizations, Aequationes Math. 31 (1986), 121-141.

[22] C. Gagliardi, Extending the concept of genus to dimension n, Proc. Amer. Math. Soc. 81 (1981), 473-481.

[23] R. Gurau, Random Tensors, Oxford University Press, 2016.

[24] R. Gurau, The complete 1/N expansion of a SYK-like tensor model, Nucl. Phys. B 916 (2017), $386-401$.

[25] J. Harer - A. Kas - R. Kirby, Handlebody decompositions of complex surfaces, Mem. Amer. Math. Soc. 62 (1986), no. 350.

[26] R. Kirby, The topology of 4-manifolds, Lecture Notes in Math. 1374, Springer-Verlag, 1989.

[27] R. Kirby, Problems in low dimensional manifold theory, Algebraic and geometric topology (Proc. Sympos. Pure Math., Stanford Univ., Stanford, Calif., 1976), Part 2, pp. 273-312, Proc. Sympos. Pure Math., XXXII, Amer. Math. Soc., Providence, R.I., 1978.

[28] R. Kirby (ed.), Problems in Low-dimensional Topology AMS/IP Stud. Adv. Math. 2(2), Geometric topology (Athens, GA, 1993), 35-473 (Amer. Math. Soc. 1997).

[29] F. Laudenbach - V. Poenaru, A note on 4-dimensional handlebodies, Bull.Soc. Math. France, 100 (1972), 337-344.

[30] R. Mandelbaum, Four-dimensional topology: an introduction, Bull. Amer. Math. Soc. 2 (1980), $1-159$.

[31] J.M. Montesinos Amilibia, Heegaard diagrams for closed 4-manifolds In: Geometric topology, Proc. 1977 Georgia Conference, Academic Press (1979), 219-237. [ISBN 0-12-158860-2]

[32] B.S. Trace, A class of 4-manifolds which have 2-spines, Proc. Am. Math. Soc. 79 (1980), 155-156.

[33] B.S. Trace, On attaching 3-handles to a 1-connected 4-manifold, Pacific J. Math. 99 (1) (1982), 175-181.

[34] E. Witten, An SYK-like model without disorder, Journal of Physics A: Mathematical and Theoretical 52 (47) 474002 (2019). 